\title{
The collapse effect and delay of reinforcement with amygdalectomized rats
}

\author{
DAVID T. GOOMAS and MARIANNE K. STEELE \\ The University of Texas Health Science Center at Dallas, Dallas, Texas 75235
}

\begin{abstract}
In Phase 1, alleyway run speeds of amygdalectomized rats given large (12 pellets) or small (2 pellets) magnitude of reward were initially faster for the large-magnitude group, with the small-magnitude group catching up (termed the collapse effect) early in training. The collapse effect for nonlesion groups given similar magnitudes of reward occurred also, but much later in training. In Phase 2, all animals were given the same number of pellets as in Phase 1 with the addition of a 60 -sec goalbox delay of reinforcement. In the lesion animals, the brief increase in performance, due to such a shift, occurred later in training than it did for the nonlesion animals. Following the brief increase there was a large decrement in responding for all groups, but the performance decrement of the lesion groups combined was less than it was for the nonlesion groups combined. Thus, rats with amygdala lesions were less responsive to original, and to changes in, contingencies of reinforcement relative to control animals. The normal function of the amygdala is briefly discussed.
\end{abstract}

The amygdaloid complex has recently been implicated in the successful behavioral adaptation of positive and negative emotional aspects of contingencies of reinforcement (Richardson, 1973). Amygdaloid lesions in rats attenuate the response-rate increase or decrease following quantitative increases or decreases, respectively, in the magnitude of reward. Below are three common examples.

Behavioral contrast, which is evoked by maintaining the reinforcement schedule constant in one component while decreasing the density of reinforcement in the other component, thereby producing response rate increases in the unchanged component (e.g., Reynolds, 1961), is not obtained with amygdalectomized rats as it is with control subjects (Henke, 1972, 1973; Henke, Allen, \& Davison, 1972).

Alleyway studies show that after some number of reinforcements in the first goalbox of a "double alley," rats run faster in the second alley on trials in which reinforcement is omitted from the first goalbox than they do on trials in which reinforcement is given (Amsel \& Roussel, 1952). The increased vigor of responses following nonreinforcement, termed the frustration effect, is not obtained, however, with amygdalectomized rats (Henke, 1977; Henke \& Maxwell, 1973).

Finally, amygdalectomized rats are less responsive to increases and decreases in magnitude of reward, as well as more resistant to extinction, than are control animals, this in a single alleyway (Kemble \& Beckman, 1970).

The authors would like to thank Donna M. Allgood for assistance provided in multiple ways. The electronic circuitry was constructed by Jack Moore. Requests for reprints should be sent to David T. Goomas, P.O. Box 521, Duncanville, Texas 75137.
All these studies, then, examine the response rate increase or decrease following changes in contingencies of reinforcement once a baseline of performance has been established.

Recently, Goomas, Hamm, and Skinner (1980) reported response rates during original contingencies of reinforcement. In that study, lesion and control animals were given either large or small magnitude of reward. This sort of design was prompted by earlier studies which had reported that when two groups of naive rats were administered either a large or a small magnitude of reward, the large-reward group ran faster in training. Later in training, however, these differences disappeared (termed the collapse effect). The small-magnitude-of-reward group reached the same asymptotic level of performance as the largereward group (Campbell, Batsche, \& Batsche, 1972; McCain, Dyleski, \& McElvain, 1971; McHose \& Moore, 1976). Goomas et al. (1980) reported that early in training, at 20 trials, the lesion group receiving small magnitude of reward caught up with the lesion group receiving large reward. The control subjects at this point in training exhibited the performance differences that intact animals usually show-the control group receiving large magnitude of reward ran reliably faster than the control group receiving small reward.

The fact that the lesion groups exhibited the collapse effect early in training while the control animals did not, suggests that lesioned animals are less responsive to the original contingencies of reinforcement. This is consonant with Richardson's (1973) theory, which, as indicated earlier, focuses primarily on the decreased responsivity of amygdalectomized animals following changes in contingencies of reinforcement. 


\section{PHASE 1}

The first portion of this experiment was an attempt to find support for the results obtained by Goomas et al. (1980).

\section{Method}

Subjects. Twenty-six experimentally naive 90 -day-old female rats of the Wistar strain, weighing 240-260 $\mathrm{g}$ at the time of surgery, were used. The subjects were housed in an air-conditioned, wellventilated room and had access to water at all times.

Surgery and Histology. Under methahexitol anesthesia (60 $\mathrm{mg} / \mathrm{kg}$ ), bilateral electrolytic lesions were placed in the amygdaloid complex of 14 rats by passing $2.0 \mathrm{~mA}$ anodal dc through the uninsulated tip of an 18-ga wire. The electrode was placed $2.0 \mathrm{~mm}$ posterior to bregma, $4.25 \mathrm{~mm}$ lateral to the midline, and $8.25 \mathrm{~mm}$ ventral to the surface of the cortex (head horizontal). Twelve rats sustained identical control operations, but no current was passed through the electrode.

At the conclusion of the experiment, the amygdalectomized animals, under deep anesthesia, were perfused with isotonic saline followed by $10 \%$ Formalin. Removed from the skull, the brains were fixed in $10 \%$ Formalin for $48 \mathrm{~h}$. Sections ranging from 25 to $75 \mu$ were mounted and stained with cresyl violet.

Apparatus. The white plywood alleyway consisted of a startbox $(32.3 \times 10.4 \times 14.2 \mathrm{~cm})$, a runway $(114.0 \times 10.4 \times 14.2 \mathrm{~cm})$, and a goalbox $(28.6 \times 10.4 \times 14.2 \mathrm{~cm})$. Hinged hardware cloth covered the entire length of the apparatus. Guillotine doors separated the startbox from the runway and the runway from the goalbox. Raising the start door activated the first of two .01-sec clocks. The interruption of a photobeam located $96.0 \mathrm{~cm}$ beyond the start door stopped the first clock (run time) and started the second clock. The interruption of a second photobeam, located $32.5 \mathrm{~cm}$ beyond the first photobeam and approximately $5 \mathrm{~cm}$ inside the goalbox, stopped the second clock (goal time). An earthen foodcup (diameter $=8.0 \mathrm{~cm}$; height $=3.1 \mathrm{~cm}$ ) was attached to the distal end of the goalbox. The second guillotine door served to confine the rat to the goalbox.

Procedure. After 14 days of postoperative recovery, the subjects were placed on a 23-h food-deprivation schedule, maintained throughout the experiment. On Days 15 to 21 , the only additional manipulation was that each subject was handled for approximately 5 min daily.

On Day 22, the first training day, lesion (L) and nonlesion (NL) animals were assigned randomly to large-(LR) or small-reward (SR) conditions. On each of the daily trials, the subjects in Groups L-LR $(n=7)$ and NL-LR $(n=6)$ received 12 45-mg Noyes pellets on each trial, while subjects in Groups L-SR $(n=7)$ and NL-SR $(n=6)$ received 2 pellets on each trial. A trial was initiated by placing the subject in the startbox. After a 3-sec orientation time, the experimenter raised the start door to allow the animal to enter the runway. After reaching the goalbox and being left there long enough to consume the reward in the baited foodcup, the subject was immediately moved to the holding cage.

On Days 1 and 2 of training, each subject was given two trials per day. Thereafter, each subject was given four trials per day.

The rats were run in rotation within six squads of four animals each, followed by one squad of two animals. Squad assignment was randomized at the beginning of training, and the rats were run in the same order each day thereafter. A maximum time of $60 \mathrm{sec}$ was allowed in each section of the alley if $60 \mathrm{sec}$ was exceeded in that alley section. If an animal did not enter the goal box within $180 \mathrm{sec}$, it was gently prodded toward the goalbox.

\section{Results}

Histology. All lesions included bilateral damage to the amygdaloid complex and ventral pyriform cor- tex. The lesions generally included damage to the cortical, basal, and central nuclei. The medial nucleus was consistently spared. Anterior-posterior damage of the amygdaloid complex corresponded to the areas depicted on Plates 30 to 35, inclusive, of the stereotaxic atlas by König and Klippel (1963). A representative lesion is shown in Figure 1.

Run speeds. The results are based on transformation $\log 10(1 / \overline{\mathrm{X}}+1)$ of the run times in the run segment of the alleyway, since differences were generally larger and more reliable in the run segment.

The decreased responsivity of lesion animals to the original contingencies of reinforcement relative to control animals suggests that the collapse effect should occur earlier in acquisition training for the lesion animals than for the nonlesion animals. Examination of Figure 2 shows that, by Day 5, there were no reliable differences in running speeds between Groups L-LR and L-SR due to the effect of magnitude of reward. On the other hand, in the control groups, Groups NL-LR and NL-SR, the performance differences due to the effect of magnitude of reward were present through Day 10. Thereafter, Group NL-SR caught up with Groups L-LR, NL-LR, and L-SR, and for the remainder of this phase of acquisition training, the performance differences among all groups appeared to be negligible. An analysis of the data in Figure 2 confirms these observations. The differences due to the effect of magnitude of reward between Groups L-LR and L-SR appeared on Day 4, with the group that received the large reward (L-LR) running faster than the group that received the small reward $[t(12)=2.20, p<.05]$. From Day 5 to the end of this phase of acquisition training there were no reliable differences between the lesion groups. The differences between Groups NL-LR and NL-SR due

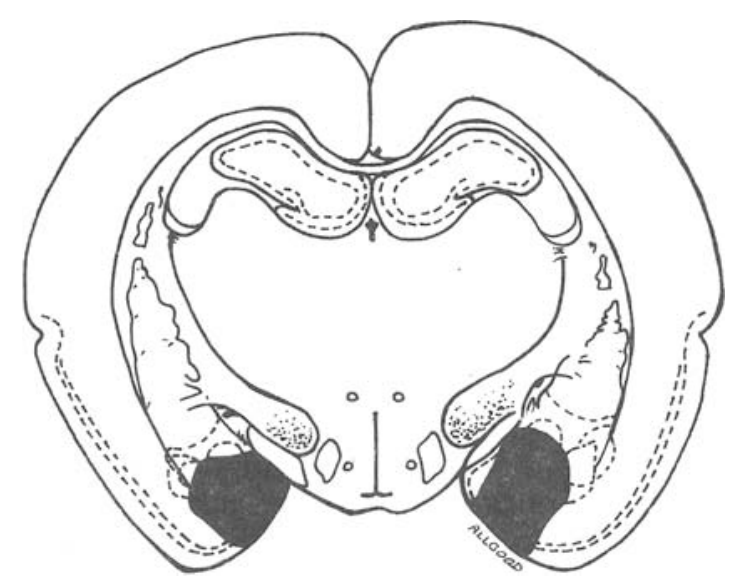

Figure 1. Representative section through the brains of an animal sustaining bilateral amygdala lesions. Cresyl violet stain. 


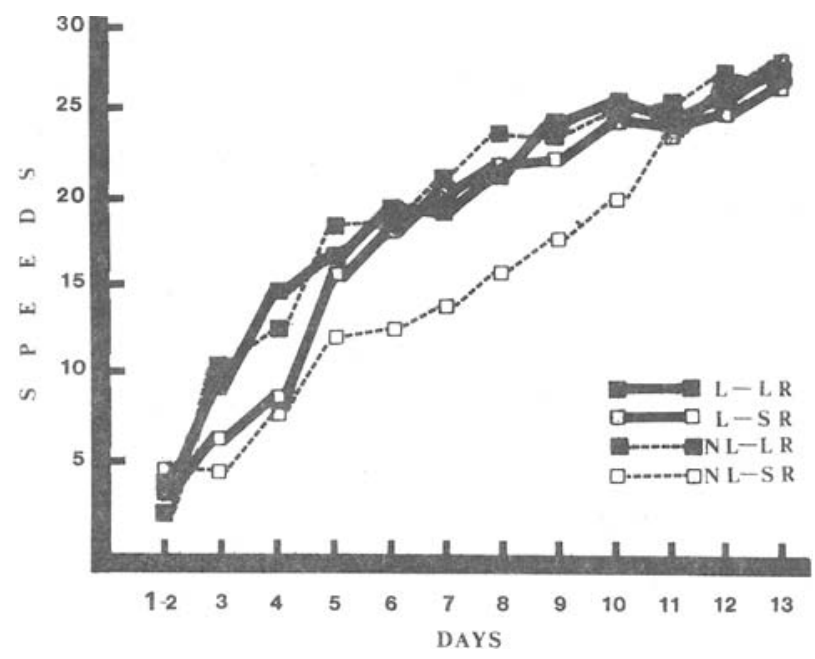

Figure 2. Run speeds of Phase 1. (Abbreviations: $\mathbf{L}=$ lesion, NL = nonlesion, $\mathbf{L R}=$ large reward, $\mathbf{S R}=$ small reward.)

to the effect of magnitude of reward appeared on Day 5 and were present through Day 10. At this time, the group receiving large reward (NL-LR) was running faster than the group given small reward (NLSR) [smallest $\mathrm{t}(10)=2.32, \mathrm{p}<.05$ ]. From Day 11 to the end of this phase of acquisition training there were no reliable differences between the nonlesion groups.

Relative performance among the groups varied as acquisition training progressed. An analysis of variance of Days 6 and 7 revealed a reliable Lesion by Reward interaction [smaller $\mathrm{F}(1,22)=5.36, \mathrm{p}<.05$ ]. Subsequent Newman-Keuls testing indicated that Groups L-LR, NL-LR, and L-SR, which did not differ among themselves $(p>.05)$, ran reliably faster than Group NL-SR $(\mathrm{p}<.05)$. On Days 12 and 13, however, the effects of lesion, reward, and their interaction were not reliable [larger $F(1,22)=.83$, $\mathrm{p}>.20$ ], indicating that there were negligible performance differences among all groups.

\section{Discussion}

The decreased responsivity in lesion animals, relative to control animals, to the original contingencies of reinforcement supports Goomas et al. (1980). While the collapse effect occurred for both lesion and control animals in acquisition training, it occurred earlier for the lesion animals.

\section{PHASE 2}

The absence or delay of a reward in a situation in which it had previously been present often produces a brief increase in performance followed by a decrease in performance (Reynolds, 1975).

In this phase of the experiment, the rats that had been given $0 \mathrm{sec}$ delay of reinforcement were shifted to a 60 -sec delay of reinforcement. Focusing on the brief increase in performance following a shift to delay of reinforcement, it seemed quite possible that (1) there would be little, if any, increased responding on the part of lesion animals relative to control animals, or (2) the increased vigor of responding exhibited by both lesion and nonlesion groups would occur later in the lesion than in the nonlesion animals in acquisition training. Note that both of these possibilities are compatible with Richardson's (1973) finding that following changes in reinforcement contingencies the responsivity of lesion animals decreases relative to that of nonlesion animals.

Kemble and Beckman (1970) reported that rats with amygdala lesions were slower than controls to extinguish a runway response. Since experimental extinction may be thought of as the limiting case of delay of reinforcement (Spence, 1956), it was predicted here that the decrement in performance resulting in a shift from immediate to delayed reinforcement would be less for the lesion than for the nonlesion animals.

\section{Method}

The subjects and apparatus were those used in Phase 1.

The subjects in this phase of training received, following a 60 -sec goalbox delay at two trials per day, 12 or 2 pellets per trial. When $60 \mathrm{sec}$ had elapsed, the experimenter baited the foodcup manually. After consuming the reward, the subject was removed immediately to the holding cage. Again, the rats were run in rotation, as in Phase 1.

\section{Results}

The results are based on transformation $\log 10$ $(1 / \bar{x}+1)$ of the run times in the run segment of the alleyway (run section data were similar to the data obtained in the goal section).

Figure 3 depicts the results of Day 13 in Phase 1 acquisition training, here labeled TA $(T A=$ terminal acquisition of Phase 1), and of the following 5 days of 60 -sec delay of reinforcement training. Figure 3 shows that the increased vigor of responding exhibited by both lesion and nonlesion groups occurred later in Phase 2 for the lesion animals (Day 2) than for the nonlesion animals (Day 1). The decrease in responding due to the shift from immediate to 60 -secdelayed reinforcement was less for the lesion animals than for the nonlesion animals. An analysis of the data in Figure 3 confirms these observations. Since increased vigor of responding is a reflection of the difference between two instances of speed, one judged to be faster than the immediately preceding instance of speed, that is, as in an ordinal scale, a nonparametric technique was used to analyze the data. Thus, tests for reliability of within-group increased vigor of responding were conducted by means of the Wilcoxon matched-pairs signed-ranks test. Progressively increasing speeds by both nonlesion groups appeared 


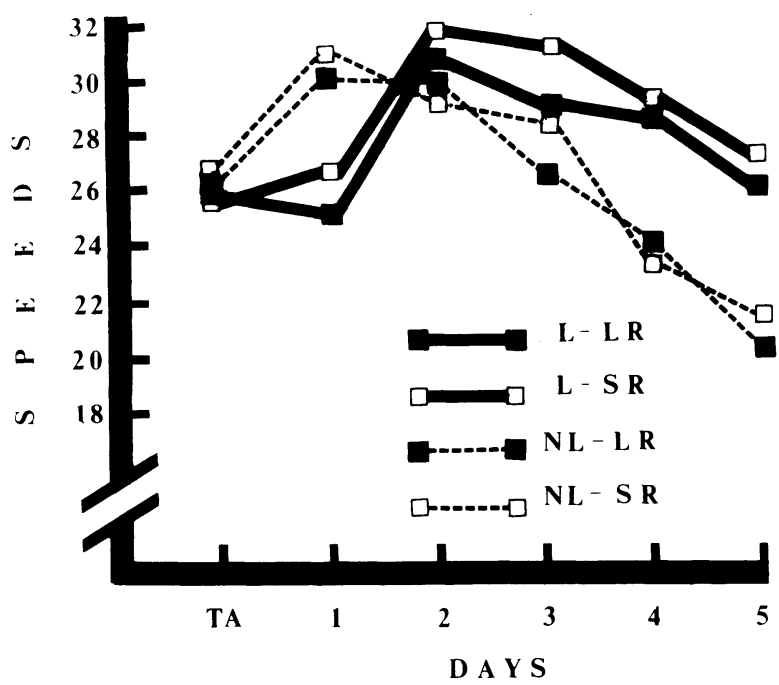

Figure 3. Run speeds of Phase 2. (Abbreviations: $\mathbf{L}=$ lesion, NL = nonlesion, $\mathbf{L R}=$ large reward, $\mathbf{S R}=$ small reward.) $R$ un speeds of Day 13 in Phase 1 acquisition training are labeled TA (terminal acquisition).

between TA and Day 1 (TA< Day 1; both Wilcoxon Ts $=0, p<.05)$, and progressively increasing speeds by both lesion groups appeared between Day 1 and Day 2 (Day $1<$ Day 2; both Wilcoxon Ts $\leqslant 1, \mathrm{p}<.05$ ). Following the brief increase in responding, the performance of all groups began to decline. An analysis of variance of Day 5 revealed a reliable lesion effect only $[F(1,22)=4.56, p<.05]$. This means that the performance decrement of the lesion groups combined was less than that of the nonlesion groups combined.

\section{Discussion}

In Phase 2, when subjects were shifted from immediate reinforcement to a 60 -sec delay of reinforcement, the lesion animals were less responsive to both the brief increase in performance and the decrease in responding that followed as a result of such a shift.

\section{GENERAL D́ISCUSSION}

This experiment indicates that amygdalectomized rats are less responsive to both original, and changes in, contingencies of reinforcement relative to control animals. A number of alleyway studies examining the food-motivated behavior of amygdalectomized rats (Goomas et al., 1980; Henke, 1977; Henke \& Maxwell, 1973; Kemble \& Beckman, 1970) now seem to suggest that the normal function of the amygdala is to interpret and integrate reinforcing stimuli. This was demonstrated electrophysiologically by Norton (1970), who reported an increase in the amplitude of the electrical activity of the amygdala of rats following rewarded barpresses but not following nonrewarded barpresses.

\section{REFERENCES}

Amsel, A., \& Roussel, J. Motivational properties of frustration: I. Effect on a running response of the addition of frustration to the motivational complex. Journal of Experimental Psychology, 1952, 43, 363-368.

Campbell, P. E., Batsche, C. J., \& Batsche, G. M. Spacedtrials reward magnitude effects in the rat: Single versus multiple food pellets. Journal of Comparative and Physiological Psychology, 1972, 81, 360-364.

Goomas, D. T., HАмm, C., \& Skinner, J. Runway performance of amygdalectomized rats: Magnitude of reinforcement and delay of food reward. Physiological Psychology, 1980, 8, 97-100.

Henke, P. G. Amygdalectomy and mixed reinforcement schedule contrast effect. Psychonomic Science, 1972, 28, 301-302.

HENKE, P. G. Effects of reinforcement omission on rats with lesions in the amygdala. Journal of Comparative and Physiological Psychology, 1973, 84, 187-193.

Henke, P. G. Dissociation of the frustration effect and the partial reinforcement extinction effect after limbic lesions in the rat. Journal of Comparative and Physiological Psychology, 1977, 91, 1032-1038.

Henke, P. G., Allen, J. D., \& Davison, C. Effect of lesions in the amygdala on behavioral contrast. Physiology \& Behavior, 1972, 8, 173-176.

Henke, P. G., \& Maxwell, D. Lesions in the amygdala and the frustration effect. Physiology \& Behavior, 1973, 10, 647-650.

Kemble, E. D., \& BeскмaN, G. J. Runway performance of rats following amygdaloid lesions. Physiology \& Behavior, $1970,5,45-47$.

König, F. R., \& KLiPPEL, R. A. The rat brain: A stereotaxic atlas of the forebrain and lower parts of the brain stem. Baltimore, Md: Williams \& Wilkins, 1963.

McCain, G., Dyleski, D., \& McElvain, G. Reward magnitude and instrumental responses: Consistent reward. Psychonomic Monograph Supplements, 1971, 3(16, Whole No. 48), 249-256.

McHose, J. H., \& Moore, J. N. Reinforcer magnitude and instrumental performance in the rat. Bulletin of the Psychonomic Society, 1976, 8, 416-418.

Norton, P. R. E. Differences in the electrophysiological correlates upon receipt of a food reward and non-reward in the rat. Brain Research, 1970, 24, 134-138.

REYNolps, G. S. Behavioral contrast. Journal of the Experimental Analysis of Behavior, 1961, 4, 57-71.

Reynolds, G. S. A primer of operant conditioning. Glenview, Ill: Scott, Foresman, 1975.

Richardson, J. S. The amygdala: Historical and functional analysis. Acta Neurobiologiae Experimentalis, 1973, 33, 623-648.

SPENCE, K. W. Behavior theory and conditioning. New Haven, Conn: Yale University Press, 1956.

(Received for publication April 29, 1980; revision accepted June 16, 1980.) 